\title{
Bursting Oscillations in Shimizu-Morioka System with Slow-Varying Periodic Excitation
}

\author{
Xindong $M a \mathbb{D}^{1,2,3}$ and Shuqian Cao $\mathbb{i D}^{1,2,3}$ \\ ${ }^{1}$ Department of Mechanics, Tianjin University, Tianjin 300354, China \\ ${ }^{2}$ Tianjin Key Laboratory of Nonlinear Dynamics and Control, Tianjin 300354, China \\ ${ }^{3}$ National Demonstration Center for Experimental Mechanics Education, Tianjin University, Tianjin 300354, China
}

Correspondence should be addressed to Shuqian Cao; sqcao@tju.edu.cn

Received 16 August 2017; Revised 23 January 2018; Accepted 4 February 2018; Published 14 March 2018

Academic Editor: Matteo Aureli

Copyright (C) 2018 Xindong Ma and Shuqian Cao. This is an open access article distributed under the Creative Commons Attribution License, which permits unrestricted use, distribution, and reproduction in any medium, provided the original work is properly cited.

\begin{abstract}
The coupling effect of two different frequency scales between the exciting frequency and the natural frequency of the ShimizuMorioka system with slow-varying periodic excitation is investigated. First, based on the analysis of the equilibrium states, homoclinic bifurcation, fold bifurcation, and supercritical Hopf bifurcation are observed in the system under a certain parameter condition. When the exciting frequency is much smaller than the natural frequency, we can regard the periodic excitation as a slowvarying parameter. Second, complicated dynamic behaviors are analyzed when the slow-varying parameter passes through different bifurcation points, of which the mechanisms of four different bursting patterns, namely, symmetric "homoclinic/homoclinic" bursting oscillation, symmetric "fold/Hopf" bursting oscillation, symmetric "fold/fold" bursting oscillation, and symmetric "Hopf/Hopf" bursting oscillation via "fold/fold" hysteresis loop, are revealed with different values of the parameter $b$ by means of the transformed phase portrait. Finally, we can find that the time interval between two symmetric adjacent spikes of bursting oscillations exhibits dependency on the periodic excitation frequency.
\end{abstract}

\section{Introduction}

Multiple time scales problems can be observed in many real systems, such as catalytic reactions in chemical systems $[1,2]$, electrophysiological experiments [3-6], and circuit systems $[7,8]$. The coupling effect of these different fastslow time scales generally results in a system that exhibits periodic motion characterized by a combination of relatively large amplitude and nearly harmonic small amplitude oscillations $[9,10]$, conventionally denoted by $N^{K}$ with $N$ and $K$ corresponding to the numbers of the large and small amplitude oscillations, respectively [11]. Variables that exhibit large amplitude oscillations generate a spiking state (SP) [12], whereas the systems may be in a quiescent state (QS) when all the variables exhibit small amplitude oscillations [13]. The dynamic behaviors connecting the spiking states and the quiescent states are called bursting oscillations. There are two types of bifurcations existing in one bursting oscillation, specifically one that transforms the system from spiking states to quiescent states and the other one that transforms the system from quiescent states to spiking states [14, 15]. Therefore, the bursting patterns not only depend on the patterns of the spiking states and quiescent states, but also depend on the patterns of the bifurcations that connect the two states [16].

For a typical system with different coupled time scales, its dynamic behaviors can be described by a singularly perturbed system with two time scales of the following form [17]:

$$
\begin{aligned}
& \dot{x}=f(x, u) \quad \text { (Fast Spiking), } \\
& \dot{u}=\varepsilon g(x, u) \quad \text { (Slow Modulation), }
\end{aligned}
$$

where $\varepsilon$ is a small parameter that satisfies $0<\varepsilon \ll 1$ and represents different time scales. Vector $x \in R^{m}$ models the dynamics of a relatively fast changing process, while vector $u \in R^{L}$ describes the relatively slowly changing quantity that modulates $x$. Characterization of the dynamic behaviors at different time scales has long intrigued scholars. Rinzel [18] introduced the fast-slow analysis method to examine the Chay-Keizer model and characterize dynamic behaviors 
at different time scales and the mechanism of bursting oscillations. Wang et al. [19] examined the synchronization of bursting through the introduction of finite delays. Kiss et al. [20] studied the bursting oscillations in electrochemical systems caused by changes in the geometrical structure. $\mathrm{Li}$ et al. [21] examined the mechanism of bursting oscillations in a nonsmooth generalized Chua's circuit with two time scales. However, most of the current work focused on the single slow variable, the mechanism of which is relatively simple, and some work is concentrated on the autonomous system. However, the mechanism of bursting oscillations in nonautonomous systems with slow-varying periodic excitation requires further exploration.

In the present work, we consider the Shimizu-Morioka system [22] with slow-varying excitation (SMSWSVE):

$$
\begin{aligned}
& \dot{x}=y \\
& \dot{y}=x-a y-x z+A \sin (\omega t) \\
& \dot{z}=-b z+x^{2},
\end{aligned}
$$

where $a \in R^{+}, b \in R^{+}, A$ is the forcing amplitude, and $\omega$ is the forcing frequency, which satisfies $0<\omega \ll 1$. When the forcing frequency $\omega$ is much smaller than the natural frequency $\omega_{0}$, we characterize the presented system as a typical fast-slow system involving two time scales. Inspired by Rinzel's method, we present an analysis of bursting oscillation driven by slow-varying periodic excitation for the ShimizuMorioka system. The purpose of this work is twofold. First, some results about bursting oscillations induced by the slow-varying periodic excitation are derived and how the excitation modulates the dynamics of bursting oscillations is drawn. Second, some important aspects of Rinzel's method are highlighted in the forced Shimizu-Morioka system.

The rest of this paper is organized as follows. In Section 2, we summarize some of the results related to the stabilities and bifurcations of SMSWSVE, and based on this, we plot a twoparameter bifurcation set and obtain three SMSWSVE bifurcation diagrams following changes in parameter $b$, which exhibit typical dynamic evolution behaviors of SMSWSVE. In Section 3, we investigate the generation of bursting oscillations based on three cases of different values of the parameter $b$, of which four bursting patterns are obtained: symmetric "homoclinic/homoclinic" bursting oscillation for $b=0.65$; symmetric "fold/Hopf" bursting oscillation for $b=1.2$; symmetric "fold/fold" bursting oscillation; and symmetric "Hopf/Hopf" bursting oscillation via "fold/fold" hysteresis loop for $b=2$. In Section 4 , we focus on the effects of the excitation amplitude and frequency on the bursting oscillation. Finally, Section 5 concludes the paper.

\section{Stabilities and Bifurcations of the SMSWSVE}

In order to analyze the influence of the slowly varying excitation, we can regard the external excitation $A \sin (\omega t)$ as a slow-varying parameter $c$. When the exciting frequency $\omega$ is much smaller than the natural frequency $\omega_{0}$, during an arbitrary period $T,\left(T=2 \pi / \omega_{0}\right)$, that is, $t \in\left(t_{0}, t_{0}+\right.$ $T$ ), where $t_{0}$ is the initial timing point, the slow-varying parameter $c$ may change between $c_{A}=A \sin \omega_{0} t_{0}$ and $c_{B}=A \sin \omega_{0}\left(t_{0}+T\right)$, thereby implying that $c_{A} \approx c_{B}$, such that the parameter $c$ is kept almost constant during any arbitrary period $T$. Therefore, (2) can be considered as a generalized autonomous system, the equilibrium points of which can also be considered as generalized equilibrium points. The generalized autonomous system in (2) forms the fast subsystem, whereas $\dot{c}=A \omega \cos (\omega t)$ forms the slow subsystem.

The equilibrium point of (2) can be expressed in the form of $\operatorname{EQ}(x, y, z)=\left(x_{0}, 0, x_{0}^{2} / b\right)$ with respect to the periodic external excitation $A \sin (\omega t)$ as the parameter $c$, where $x_{0}$ satisfies

$$
x_{0}^{3}-b x_{0}-b c=0
$$

the stability of which can be determined by the corresponding characteristic equation, which is defined as

$$
\lambda^{3}+(a+b) \lambda^{2}+\left(a b+\frac{x_{0}^{2}}{b}-1\right) \lambda+3 x_{0}^{2}-b=0 .
$$

Obviously, EQ is stable for $a+b>0,3 x_{0}^{2}-b>0$, and $(a+b)\left(a b+x_{0}^{2} / b-1\right)-\left(3 x_{0}^{2}-b\right)>0$. When the stability conditions are changed, different types of bifurcations may occur as follows.

Fold Bifurcation. For $3 x_{0}^{2}-b=0$, the three equilibrium points may meet together to form one degenerate equilibrium point, thereby generating eigenvalues for the characteristic equation that can be expressed as $\lambda_{1}=0$, and $\lambda_{2,3}=(-(a+b) \pm$ $\left.\sqrt{(a+b)^{2}-4\left(a b+x_{0}^{2} / b-1\right)}\right) / 2$, which imply the presence of fold bifurcation.

Hopf Bifurcation. $(a+b)\left(a b+x_{0}^{2} / b-1\right)-\left(3 x_{0}^{2}-b\right)=0$ defines the presence of a pair of pure imaginary eigenvalues, which implies the occurrence of supercritical Hopf bifurcation and thus results in periodic oscillation with a frequency of $\Omega_{H}=$ $\sqrt{a b+x_{0}^{2} / b-1}$.

Here we fix the parameter $a=1$ and regard the parameters $b$ and $c$ as the control parameters. The two bifurcation sets of SMSWSVE are plotted in Figure 1, wherein the parameter plane $(c, b)$ is divided into eight regions. According to our numerical calculations, region 8 exhibits an unstable equilibrium point $E_{0}$ and two stable equilibria $E_{ \pm}$. When the parameters pass through the fold bifurcation curves LP1 and LP2 and enter regions 7 and 6, the numbers of the equilibria may decrease via fold bifurcation, thereby leaving two stable equilibria $E_{+}$in region 7 and $E_{-}$in region 6. As the parameters pass through the Hopf bifurcation curves supH1 and supH2 and enter regions 5 and 4, the two stable equilibria, specifically $E_{+}$and $E_{-}$, lose their stabilities via Hopf bifurcations and are left with two stable limit cycles, namely, LC1 in region 5 and LC2 in region 4 . When the parameters pass through the fold bifurcation curves LP1 and LP2 and enter regions 3 and 2, the two stable limit cycles LC1 


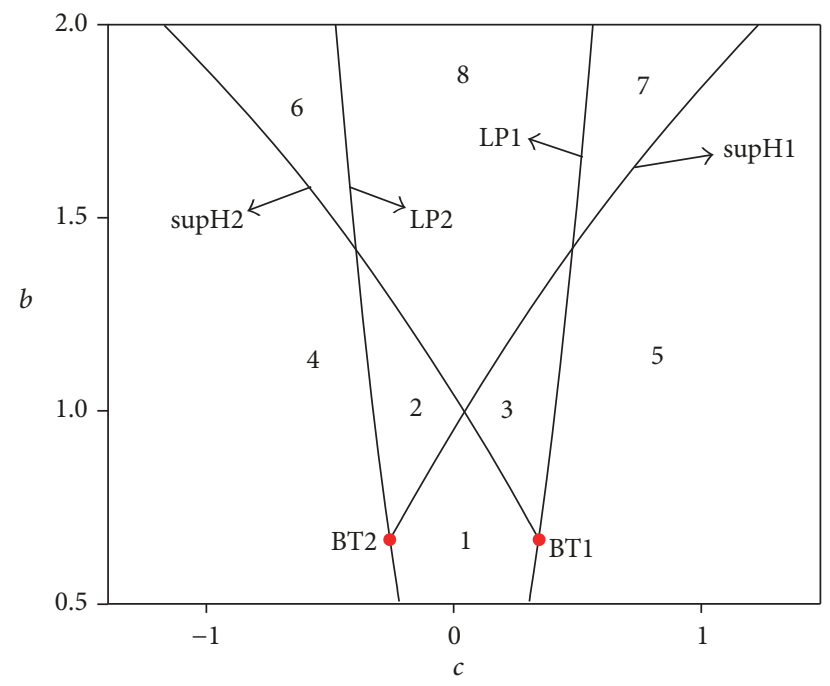

Figure 1: Two bifurcation sets of the system with respect to parameters $b$ and $c$, for fixed $a=1$. BT1 and BT2 represent the two Bogdanov-Takens bifurcation points; LP1 and LP2 represent the twofold bifurcation curves; and supH1 and supH2 represent the two Hopf bifurcation curves.

and LC2 are still observed, and the numbers of the equilibria may increase via fold bifurcation, specifically one stable equilibrium point and two unstable equilibrium points. As the parameters pass through the Hopf bifurcation curves supH1 and supH2 and enter region 1, the stable equilibrium point loses its stability, thereby producing one stable limit cycle via Hopf bifurcation. Therefore, three unstable equilibria and two stable limit cycles are observed in region 1.

From the two bifurcation sets of SMSWSVE with respect to the parameters $b$ and $c$, we can find that when the parameter $b$ is taken at different values, the two types of curves corresponding to fold bifurcation and Hopf bifurcation exhibit different positions. For example, when the parameter $b$ is smaller than 0667, no Hopf bifurcation is observed. When the parameter $b$ is greater than 0667 but smaller than 1.420, the supH1 curve is observed on the left side of the LP1 curve and the supH2 curve is observed on the right side of the LP2 curve, thereby indicating that a sufficiently large amplitude $A$ of the parameter $c$ generates an increase (decrease) in the parameter $c$ from point 0 , wherein it first passes through the Hopf bifurcation points and then passes through the fold bifurcation points. When the parameter $b$ is greater than 1.420 , the supH1 curve is observed on the right side of the LP1 curve and the supH2 curve is observed on the left side of the LP2 curve, thereby indicating that the absolute value of the Hopf bifurcation points is greater than the absolute value of the fold bifurcation points.

To comprehensively investigate the influence of the slowvarying periodic excitation, three cases with different values of the parameter $b$, that is, case $\mathrm{A}: b=0.65$, case $\mathrm{B}: b=1.2$, and case $C: b=2.0$, are considered, the bifurcations of which are presented in Figure 2, respectively.
For $b=0.65$ in Figure 2(a), twofold bifurcation points are observed at LP1 $(0.310,-0.466)$ and LP2 $(-0.310,0.466)$. And two homoclinic bifurcation points are observed at Homl $(0.029,-0.034)$ and Hom2 $(-0.029,0.034)$, at which the two stable limit cycles can be switched.

For $b=1.2$ in Figure 2(b), twofold bifurcation points are observed at LP1 $(0.420,-0.640)$ and LP2 $(-0.420,0.640)$, whereas two supercritical Hopf bifurcation points are observed at supH1 $(0.203,1.187)$ and supH $2(-0.203,-1.187)$, at which the two limit cycles are produced via Hopf bifurcation.

For $b=2.0$ in Figure 2(c), twofold bifurcation points are observed at LP1 $(0.539,-0.806)$ and LP2 $(-0.539,0.806)$, at which the jumping phenomenon may occur. In addition, two supercritical Hopf bifurcation points are observed at supH1 $(1.222,1.834)$ and supH2 $(-1.222,-1.834)$, at which the two limit cycles are produced via Hopf bifurcation.

Since $c=A \sin (\omega t)$, the change interval of the parameter $c$ depends on its amplitude $A$. Therefore, the influence of the amplitude $A$ on the system can be examined to characterize the dynamic behaviors of SMSWSVE when $c$ passes through different bifurcation points.

\section{Dynamical Mechanisms of the Bursting Oscillations}

The present section characterizes the dynamical behaviors of (2) for the three typical cases. In this article, SMSWSVE is a typical fast-slow system involving two time scales, which may generate bursting oscillations in the system. In the following analysis, the excitation frequency is fixed at $\omega=0.005$.

3.1. Symmetric "Homoclinic/Homoclinic" Bursting in Case A. In case $\mathrm{A}$ for $b=0.65$, no stable equilibrium points are observed. However, one stable limit cycle LC1 is observed for $c>-0.029$, one stable limit cycle LC2 is observed for $c<0.029$, and two stable limit cycles are observed for $-0.029<c<0.029$ simultaneously. The stable limit cycle LC1 disappears via homoclinic bifurcation of the saddle point at Hom $2(-0.029,0.034)$, and the limit cycle LC2 disappears via homoclinic bifurcation of the saddle point at Hom1 $(0.029,-0.034)$. The two stable limit cycles can be switched via two homoclinic bifurcation points, namely, Hom1 and Hom2.

Figure 3 presents the bursting oscillation for $A=0.3$, wherein the trajectory moves around two stable limit cycles, namely, LC1 and LC2, as presented in Figure 3(a), which are switched by the two homoclinic bifurcation points. In order to reveal the mechanism of this bursting oscillation, we turn to the help of the transformed phase portrait on the $(c, x)$ plane, as presented in Figure 3(b). The trajectory, starting from the point $A$, moves along the attraction domain of the stable limit cycle LC1, and the movement exhibits a repetitive spiking state with large amplitude. As the parameter $c$ decreases to -0.029 , where the homoclinic bifurcation point Hom2 is located, LC1 disappears via homoclinic bifurcation. In addition, the trajectory jumps into the vector field of 


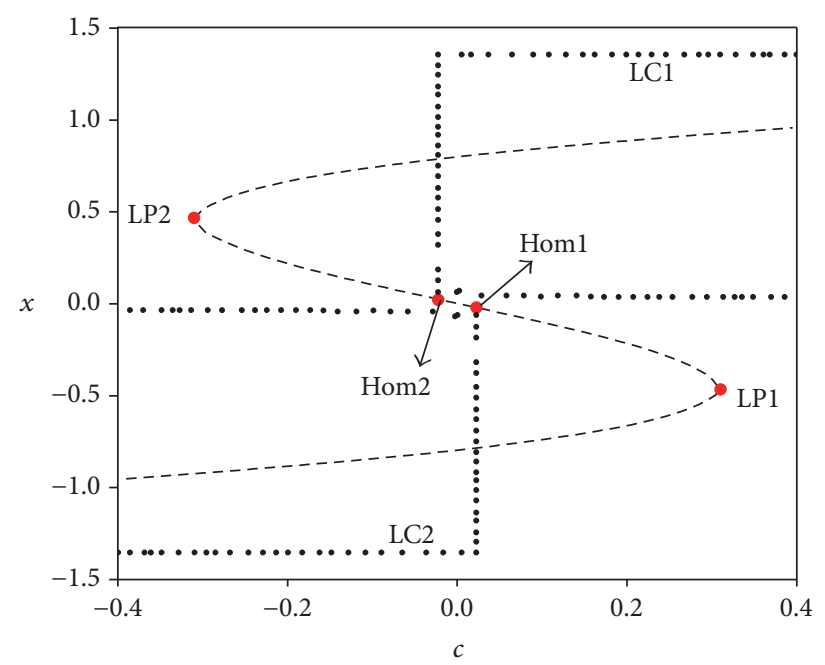

(a)

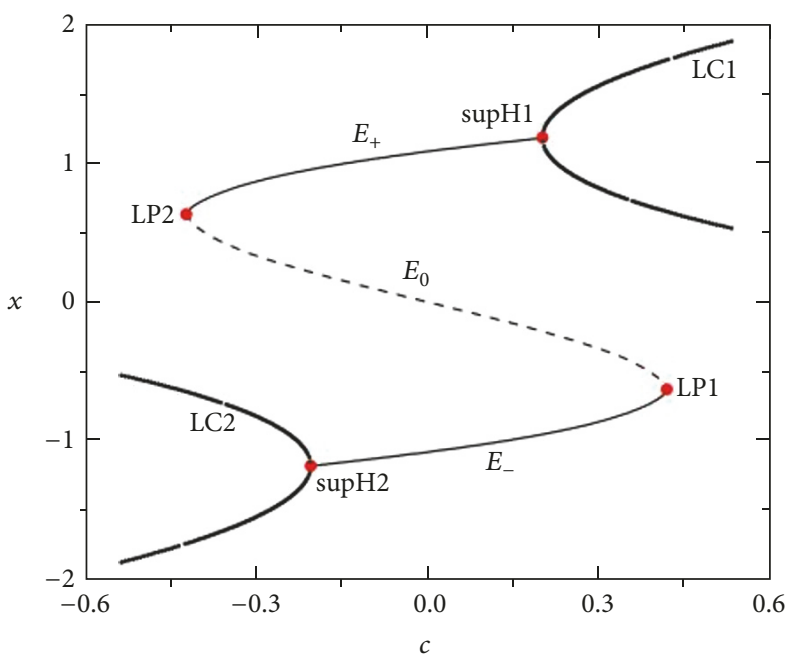

(b)

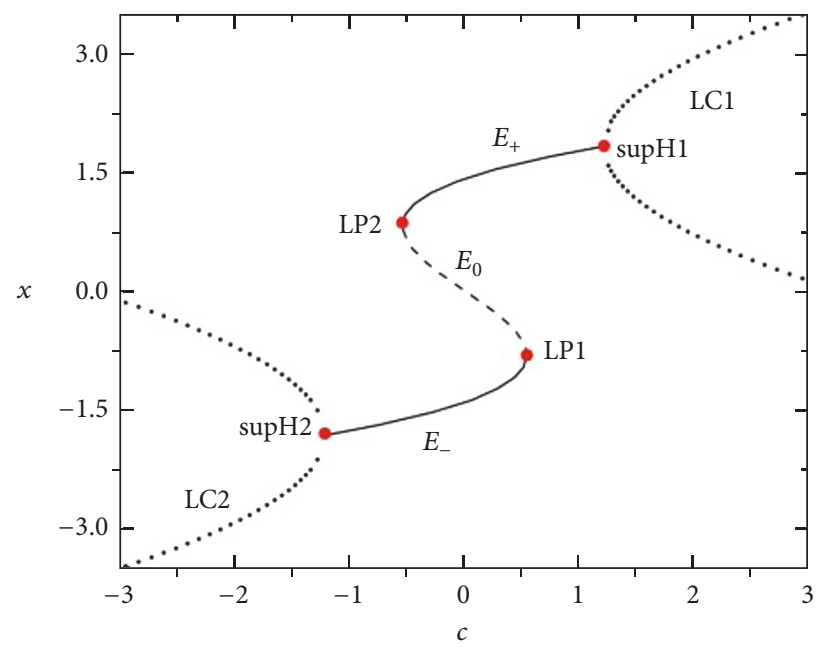

(c)

FIGURE 2: Bifurcation diagrams for (a) $b=0.65$; (b) $b=1.2$; and (c) $b=2$. The solid line represents the stable parts; the dashed line represents the unstable parts; represents the stable limit cycles; Homl and Hom2 represent the two homoclinic bifurcation points; LP1 and LP2 represent the twofold bifurcation points; supH1 and supH2 represent the two Hopf bifurcation points; $\mathrm{LC1}$ and LC2 represent the two stable limit cycles; and $E_{+}, E_{-}$, and $E_{0}$ represent the equilibrium branch of the generalized autonomous system.

stable limit cycle LC2. Parameter $c$ continues to decrease until it reaches its minimum value of $c=-0.3$, after which it gradually increases.

When the parameter $c$ increases to 0.029 , where the homoclinic bifurcation point Hom1 is located, LC2 disappears via saddle homoclinic bifurcation, and the trajectory jumps into the vector field of the stable limit cycle LC1. Parameter $c$ continues to increase until it reaches its maximum value of $c=0.3$, after which it gradually decreases. The return of the trajectory to starting point $A$ finished one periodic movement.

Bursting oscillation is observed obviously in the time history in Figure 3(c), wherein the up-state and the down-state are switched via the two homoclinic bifurcation points that form the bursting oscillation with the symmetric structure. Therefore, the bursting can be classified as the symmetric "homoclinic/homoclinic" type.
3.2. Symmetric "Fold/Hopf" Bursting in Case B. In case B for $b=1.2$, twofold bifurcation points LP1, $2( \pm 0.420, \pm 0.640)$ and two Hopf bifurcation points supH1, $2( \pm 0.203, \pm 1.187)$ are observed on the $(c, x)$ plane, which result in the transition between the repetitive spiking and quiescent states.

When the amplitude $A$ is taken at $A=0.430$, the slow variable $c$ exhibits changes between -0.430 and 0.430 , that is, $c \in[-0.430,0.430]$, thereby implying the presence of the fold bifurcation points at $c= \pm 0.423$ and the Hopf bifurcation points at $c= \pm 0.203$. From the phase portrait on the $(x, y)$ plane plotted in Figure 4(a), the attractors oscillate around two equilibrium points, as denoted by $E_{ \pm}$. In addition, a typical bursting oscillation is observed in the time history in Figure 4(b). The trajectory jumps between two spiking states $\mathrm{SP}_{ \pm}$, which are symmetric to each other.

In order to reveal the mechanism of the bursting oscillation, we turn to the transformed portrait phase with 


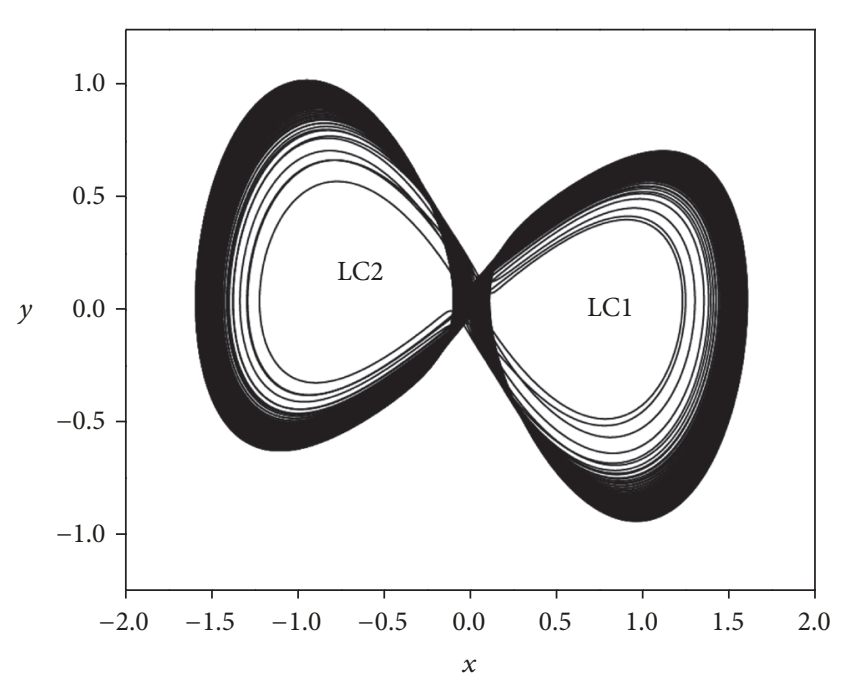

(a)

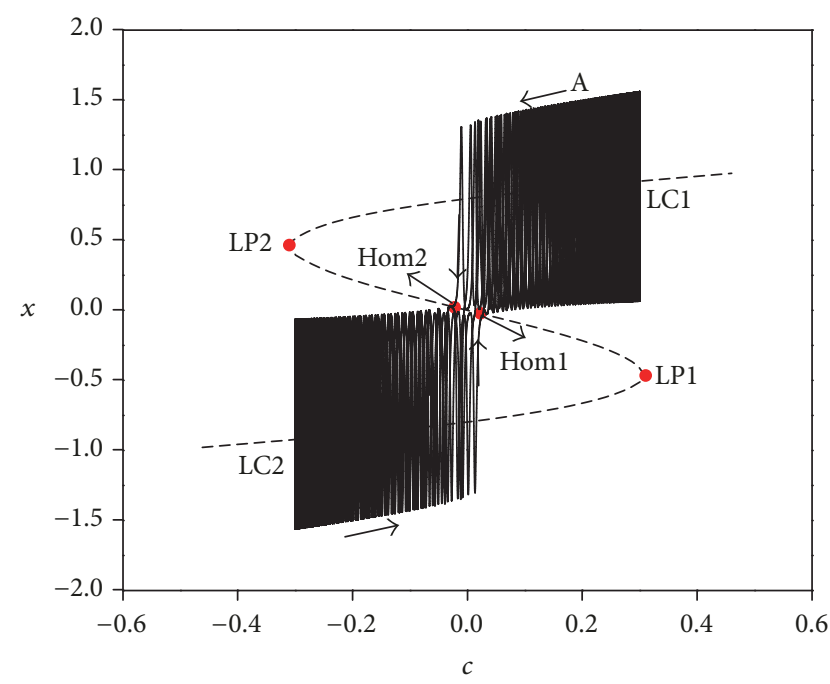

(b)

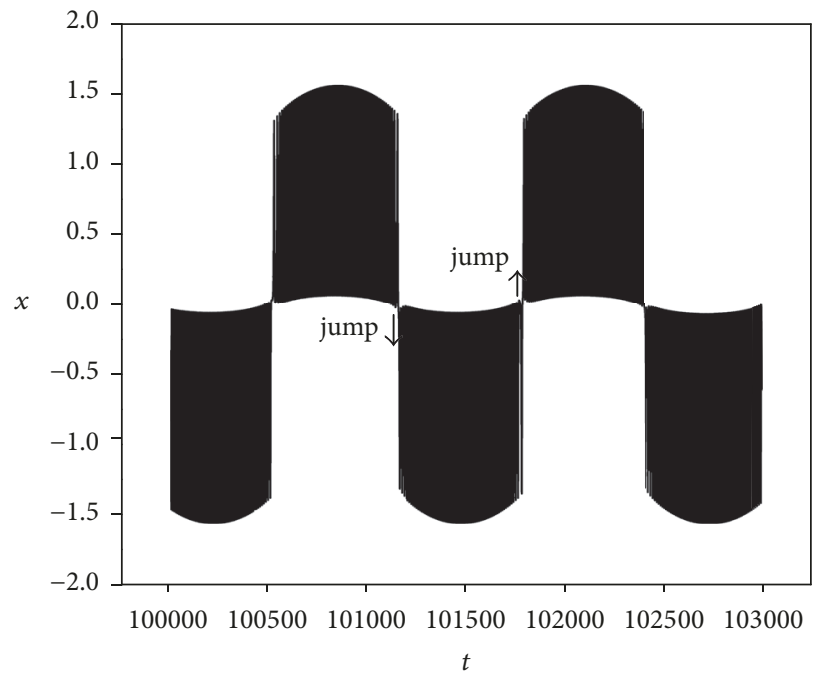

(c)

Figure 3: Bursting oscillation for $A=0.3$. (a) Phase portrait on the $(x, y)$ plane; (b) transformed phase portrait on the (c, $x)$ plane with equilibrium branches; (c) time history.

the bifurcation diagram, as presented in Figure $4(\mathrm{c})$. The trajectory starts from the point $A$ with $c=0.430$, where $c$ is taken at its positive extreme value. At this point, the attractor is categorized as the stable limit cycle LC1, thereby allowing the trajectory to move within the vector field of LC1, and repetitive spiking movement with large amplitude is observed. Parameter $c$ then decreases, as indicated by the arrow. When the parameter $c$ decreases to $c=0.203$, where the Hopf bifurcation point supH1 is observed, thereby resulting in the disappearance of stable limit cycle LC1 and the appearance of stable equilibrium point $E_{+}$.

Due to the slow passage effect [23], the trajectory will not move within the vector field of $E_{+}$immediately, it still moves in the vector field of LC1 for a short time, and then it turns into the vector field of $E_{+}$. The repetitive spiking state movement is terminated, and the quiescent state movement is created. Parameter $c$ then continues to decrease to $c=-0.420$, where the fold bifurcation point LP2 is located, thereby generating a trajectory jump into the vector field of LC2 and resulting in the termination of the quiescent state movement and the creation of repetitive spiking state movement. As the parameter $c$ decreases to $c=-0.430$, it reaches its negative extreme value, after which the parameter $c$ exhibits a gradual increase.

When the parameter $c$ increases to $c=-0.203$, where the other Hopf bifurcation point supH2 is observed, it results in the disappearance of the stable limit cycle LC2 and the appearance of the stable equilibrium point $E_{-}$. The trajectory still moves in the vector filed of LC2 for a short time according to the slow passage effect, after which it turns into the vector field of $E_{-}$. The Hopf bifurcation point supH2 terminates the repetitive spiking state movement and exhibits the quiescent 


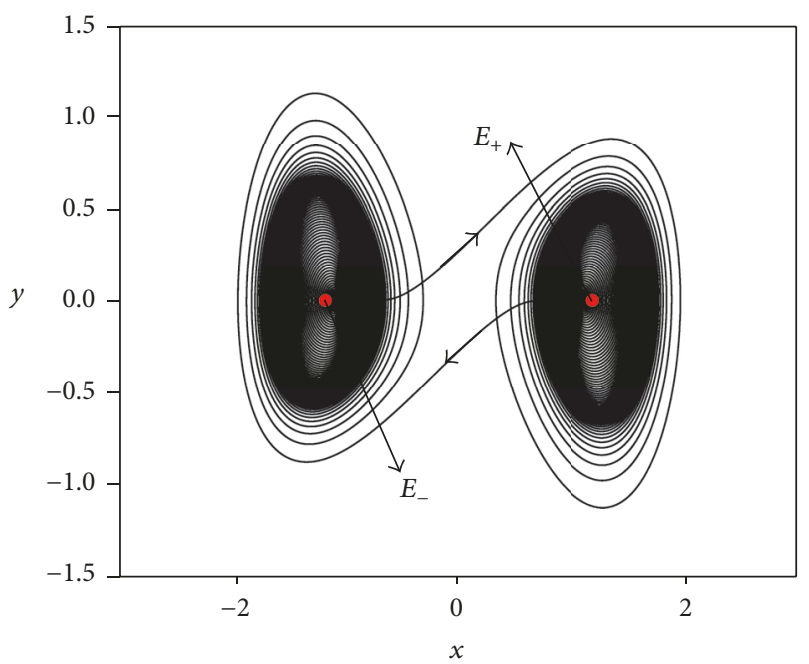

(a)

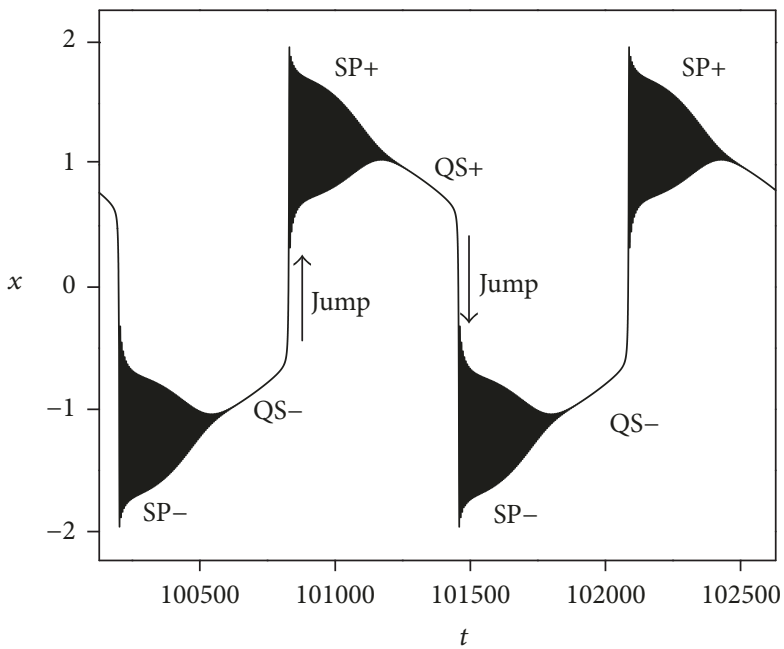

(b)

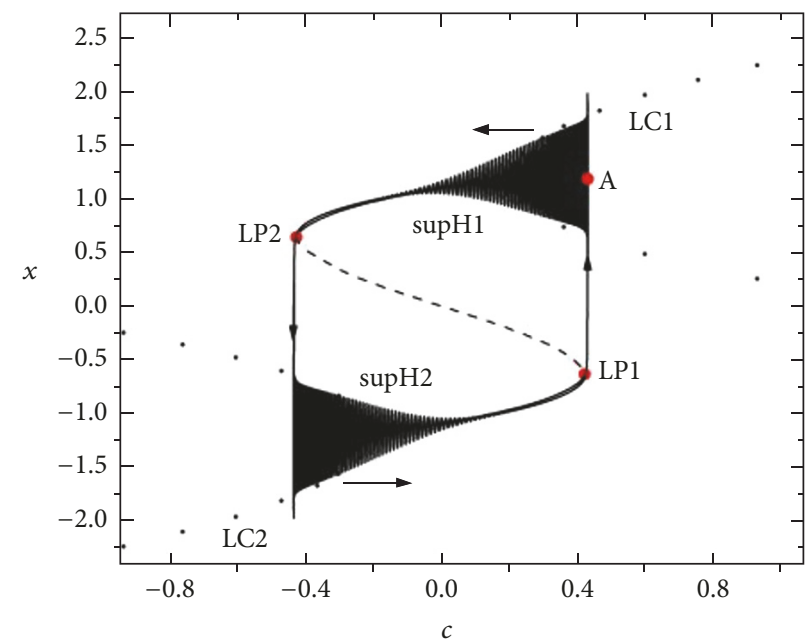

(c)

FIgURE 4: Bursting oscillation for $A=0.430$. (a) Phase portrait on the $(x, y)$ plane; (b) time history; (c) transformed phase portrait on the $(c, x)$ plane with the bifurcation diagram.

state movement. When the parameter $c$ increases to $c=$ 0.420 , where the fold bifurcation point LP1 is observed, it results in the disappearance of the quiescent state movement and the appearance of the repetitive spiking state movement. In addition, this increase results in the loss of $E_{-}$'s stability and the generation of the stable limit cycle LC1. Parameter $c$ then continues to increase to $c=0.430$. The return of the trajectory back to the starting point $A$ finished one periodic movement.

The bursting oscillation can be classified as symmetric "fold/Hopf" bursting, since the repetitive spiking related to the limit cycle is generated by fold bifurcation and disappears by Hopf bifurcation.

3.3. Bursting Oscillation in Case $C$. In case $\mathrm{C}$ for $b=$ 2 , twofold bifurcation points LP1, $2( \pm 0.539, \pm 0.806)$ and two Hopf bifurcation points supH1, $2( \pm 1.222, \pm 1.834)$ are observed on the $(c, x)$ plane, resulting in the transition between the repetitive spiking and quiescent states. Two different types of bursting oscillations are observed when the amplitude $A$ is taken at different values.

3.3.1. Symmetric "Fold/Fold" Bursting Oscillation. When the amplitude $A$ is taken at $A=1.0$, the slow variable $c$ changes between -1.0 and 1.0, thereby implying the presence of only twofold bifurcation points at $c= \pm 0.539$. Under this condition, three equilibria are present: two stable foci denoted by $E_{ \pm}$and one unstable saddle point denoted by $E_{0}$, as presented in Figure 5.

From the phase portrait on the $(x, y)$ plane, as presented in Figure 6(a), the trajectory oscillates around two equilibrium points, namely, $E_{ \pm}$. A typical bursting oscillation is observed in the time history diagram in Figure 6(b). In 


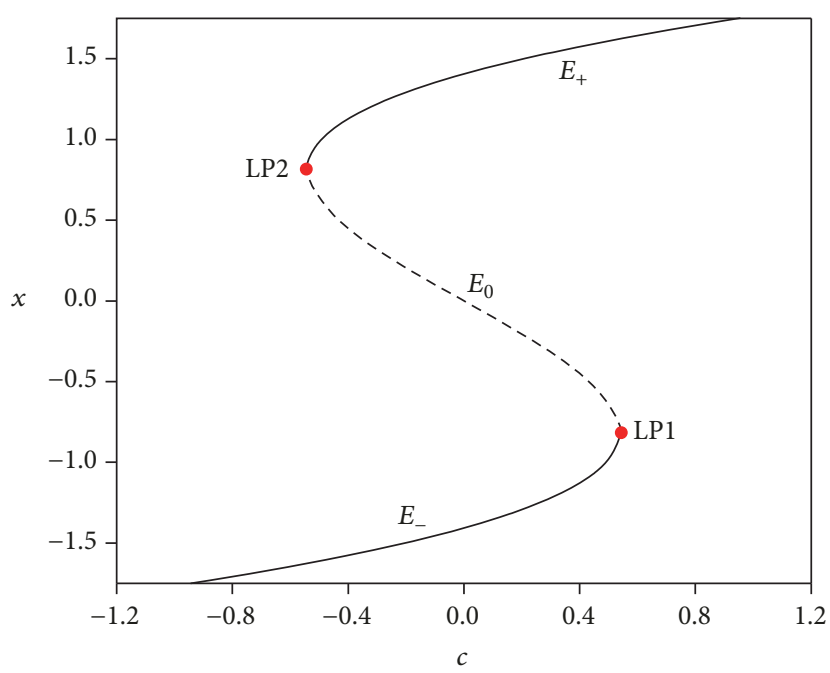

FIGURE 5: Distribution of the equilibria for $A=1.0$.

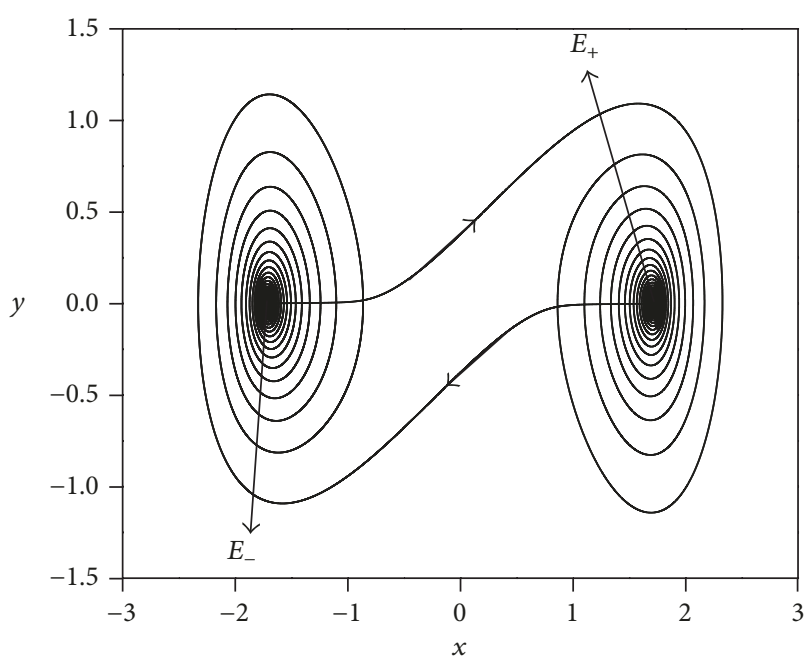

(a)

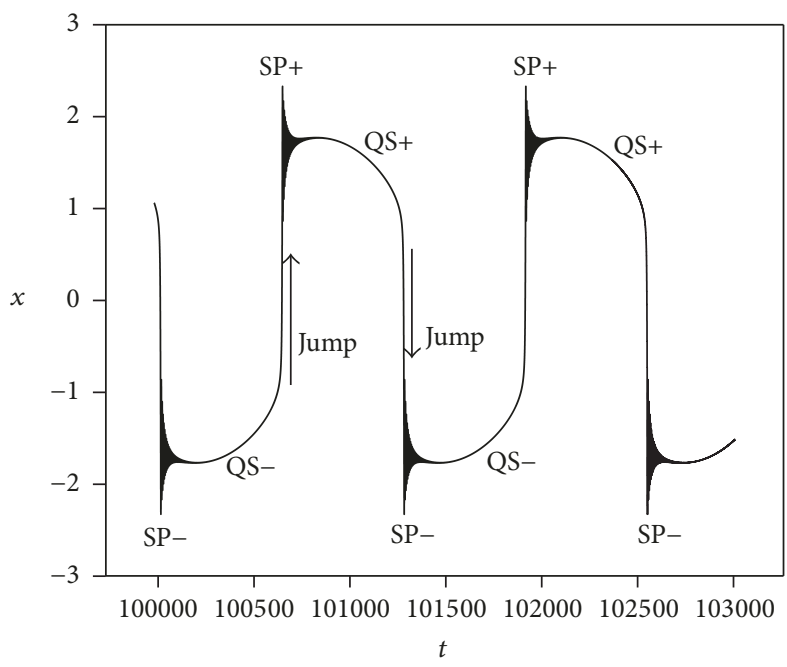

(b)

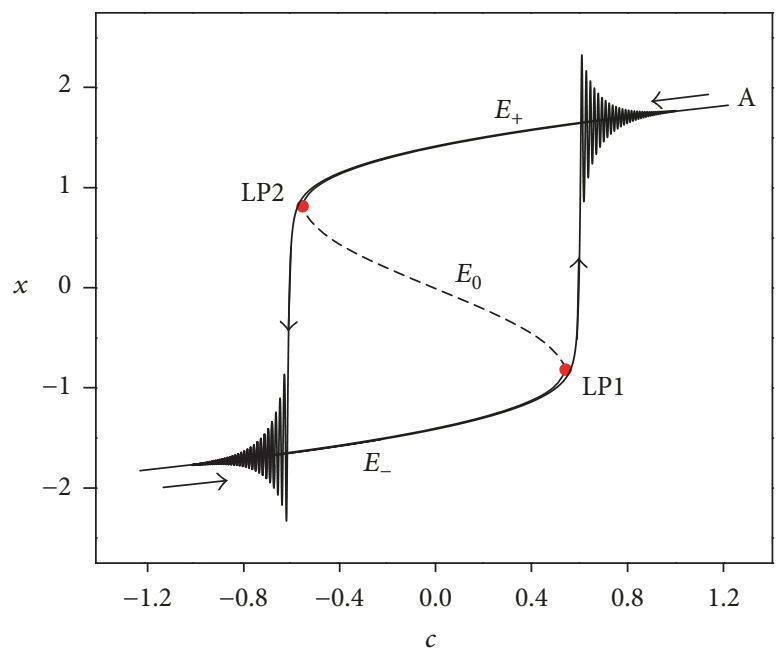

(c)

Figure 6: Bursting oscillation for $A=1.0$. (a) Phase portrait on the $(x, y)$ plane; (b) time history; (c) transformed phase portrait on the ( $c$, $x$ ) plane with equilibrium branches. 
addition, the trajectory jumps between two spiking states, specifically $\mathrm{SP}_{ \pm}$, which are symmetrical to each other.

In order to reveal the mechanism of the bursting oscillation, we turn to the transformed phase portrait on the $(c, x)$ plane with equilibrium branches, as presented in Figure 6(c). The trajectory starts from the point $A$ with $c=1.0$, where the parameter $c$ is taken at its positive extreme value. The trajectory moves within the vector field of the stable focus $E_{+}$that is associated with the quiescent state. When the parameter $c$ decreases to $c=-0.539$, where the fold bifurcation point LP2 is observed, thereby resulting in a trajectory jump to the vector field of the other stable focus $E_{-}$and ending the quiescent state. The trajectory exhibits high-frequency oscillation with a gradual decrease in the amplitude, which corresponds to the spiking state. As the parameter $c$ decreases to its negative value, specifically $c=-1$, it may generate a gradual increase that corresponds to the quiescent state.

When the parameter $c$ increases to $c=0.539$, where the fold bifurcation point LP1 is observed, it results in a trajectory jump to the vector field of $E_{+}$and terminating the quiescent state. In addition, the trajectory is characterized as high-frequency oscillation with a gradual decreasing in the amplitude, which corresponds to the spiking state. As the parameter $c$ increases to $c=1$, the trajectory returned back to its starting point $A$, thereby finishing one periodic movement.

Bursting oscillation can be created since the system periodically switches between the two stable foci $E_{+}$and $E_{-}$ by twofold bifurcations and the two parts are symmetrical. So the bursting pattern can be classified as symmetric "fold-fold" type.

3.3.2. Symmetric "SupHopf/SupHopf" Bursting Oscillation via "Fold/Fold" Hysteresis Loop. A continues to increase to the range of $A>1.222$, for example, $A=2$, thereby implying that the parameter $c$ not only passes through the fold bifurcation points LP1 and LP2 at $c= \pm 0.539$, but also passes through the supercritical Hopf bifurcation points supH1 and supH2 at $c= \pm 1.222$, where the Hopf bifurcation occurs.

According to the phase portrait on the $(x, y)$ plane in Figure $7(\mathrm{a})$, the trajectory oscillates around two stable foci, namely, $E_{ \pm}$. In addition, a typical bursting oscillation is observed in the time history diagram in Figure $7(\mathrm{~b})$, and the trajectory jumps between two spiking states, specifically $\mathrm{SP}_{ \pm}$, both of which are symmetrical to each other. However, a great difference is observed between Figure 7(a) and Figure 6(a) because two limit cycles are observed in Figure 7(a).

In order to explore the mechanism of the bursting oscillation in Figure 7, we turn to the overlap of the transformed phase portrait on the $(c, x)$ plane with the system bifurcation diagram, as presented in Figure 7(c). We assume that the trajectory, starting from the point $A$ with $c=2$, moves within the vector field of the limit cycle $\mathrm{LC} 1$, corresponding to the spiking state. When the parameter $c$ decreases to $c=$ 1.222 , where the Hopf bifurcation point supH1 is observed, thereby resulting in the disappearance of limit cycle LC1 and the presence of the stable focus $E_{+}$. In addition, the
Hopf bifurcation point supH1 resulted in the termination of the spiking state and the appearance of the quiescent state. As the parameter $c$ decreases to $c=-0.539$, where the fold bifurcation point LP2 is observed, the trajectory jumps into the vector field of the stable focus $E_{-}$, and the fold bifurcation point LP2 also results in the termination of the quiescent state and the appearance of the spiking state. When the parameter $c$ decreases to $c=-1.222, E_{-}$loses its stability via Hopf bifurcation, and a stable limit cycle LC2 is observed. As the parameter $c$ decreases to an negative extreme value, specifically $c=-2$, it exhibits a gradual increase.

When the parameter $c$ increases to $c=-1.222, \mathrm{LC} 2$ disappears and $E_{-}$is observed due to Hopf bifurcation. In addition, this increase results in the termination of the spiking state and the appearance of the quiescent state. As the parameter $c$ increases to $c=0.539$, where the fold bifurcation point LP1 is observed, which results in the trajectory jumps to the vector field of $E_{+}$, thereby terminating the quiescent state and generating the presence of the spiking state. When the parameter $c$ exhibits an increase to $c=1.222$, where the Hopf bifurcation point supH1 is observed, $E_{+}$loses its stability and the stable limit cycle LC1 is produced via Hopf bifurcation. As the parameter $c$ increases to $c=2$, the trajectory returns back to its starting point $A$, thereby finishing one periodic movement.

From Figure 7(c), a hysteresis loop produced by the equilibrium branches between twofold bifurcation points, specifically $\mathrm{LP}_{1,2}$, is observed. As a result, this bursting is classified as symmetric "Hopf/Hopf" type via the "fold/fold" hysteresis loop.

\section{Effects of the Excitation Amplitude and Frequency on the Bursting Dynamics}

We have investigated the dynamical mechanisms of bursting oscillations. In this section, we focus on the effects of excitation amplitude and excitation frequency on the bursting dynamics.

First, the effect of the excitation amplitude on the bursting dynamics is considered. Let us take the symmetric "fold/fold" bursting oscillation in case $\mathrm{C}$ as an example. According to the phase portrait related to $c$ in Figures 6 and 7, bursting oscillation is created when the slow excitation $c$ passes through twofold bifurcation values $c_{\mathrm{LP} 1}$ and $c_{\mathrm{LP} 2}$ periodically. Since $c=A \sin (\omega t)$, if the excitation $A$ satisfies $A<$ $\left|c_{\mathrm{LP}}\right|$, the bursting oscillations are not created because the slow excitation cannot pass through the twofold bifurcation values, while if $A>\left|c_{L P}\right|$, they may be created.

Second, the effect of the excitation frequency on the bursting dynamics is considered. The three bursting patterns are symmetrical in this article. Therefore, the time interval between two adjacent spikes of bursting in one oscillation period is half a period, which is the minimum time interval $\Delta T=(1 / 2) T=\pi / \omega$ between two adjacent spikes, where $T$ is the period of one movement. Take the waveforms in Figure 7 as an example, when $\omega_{1}=0.0025, \omega_{2}=0.005$, and $\omega_{3}=0.01$, respectively, the corresponding time intervals between two 


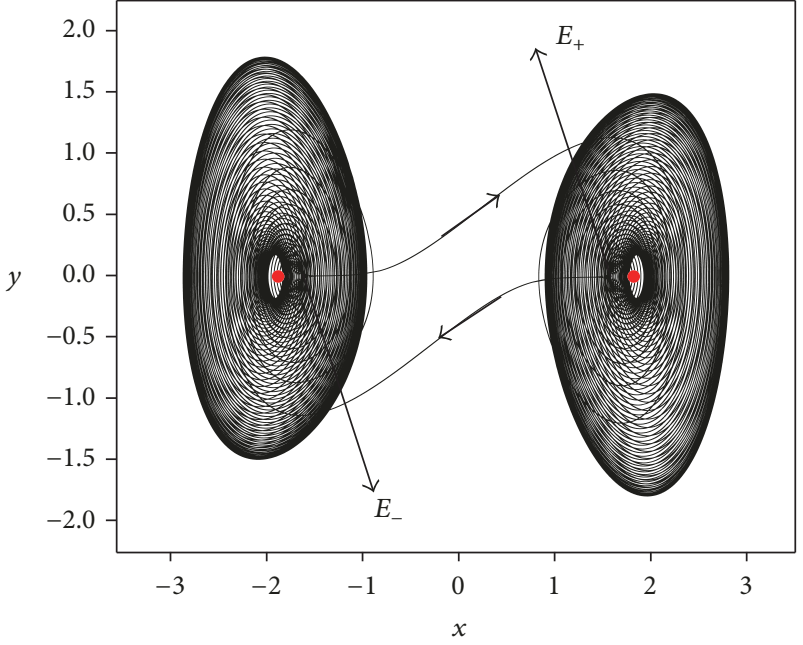

(a)

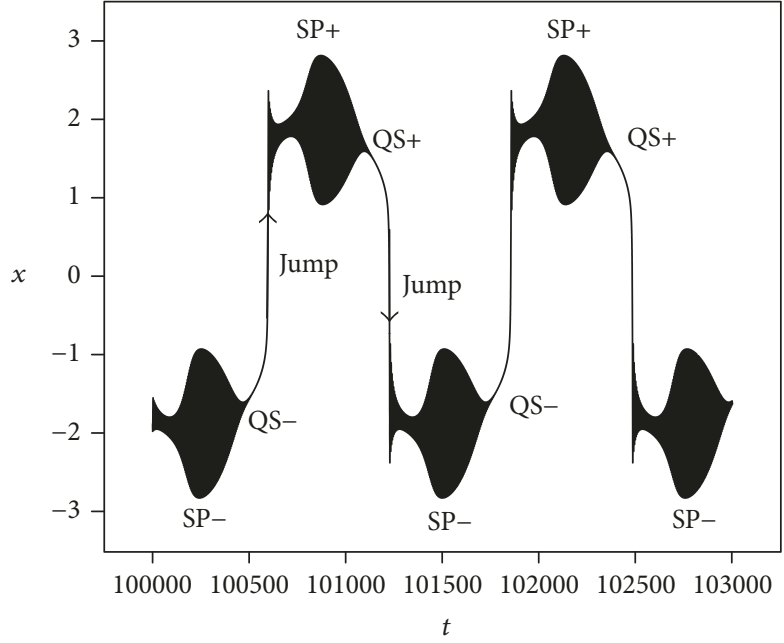

(b)

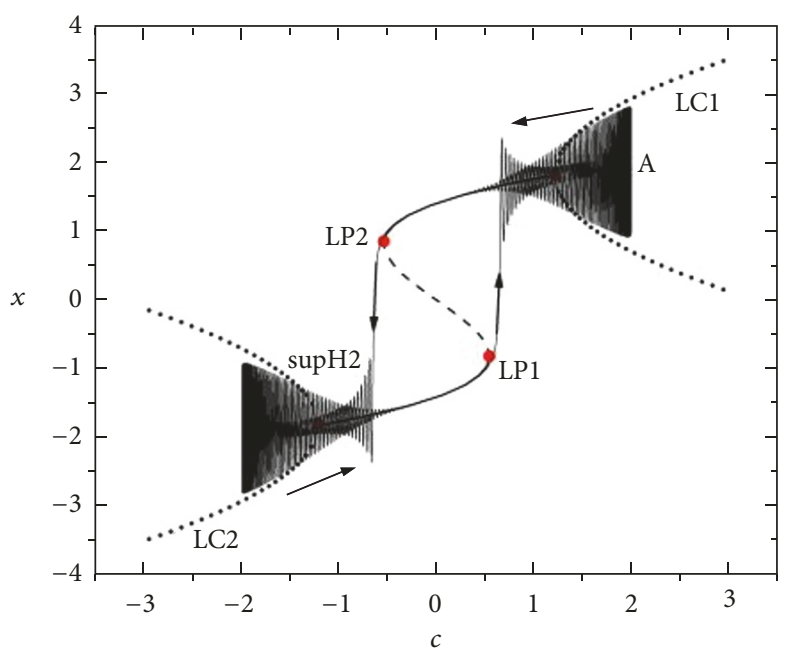

(c)

Figure 7: Bursting oscillation for $A=2$. (a) Phase portrait on the $(x, y)$ plane; (b) time history; (c) overlap of the transformed phase portrait on the $(c, x)$ plane with the system bifurcation diagram.

adjacent spikes are measured, as presented in Figure 8, of which the numerical results agreed well with the analytical ones.

\section{Conclusions}

The Shimizu-Morioka system may exhibit bursting oscillations with different waveforms under certain external forcing conditions. This article theoretically explores the mechanisms of different bursting patterns in the presence of two different frequency scales between the exciting frequency and the natural frequency. First, we regard the slow excitation $A \sin (\omega t)$ as a slow-varying parameter $c$. Second, according to the two bifurcation sets of $(c, b)$, three cases are considered: namely, $b=0.65, b=1.2$, and $b=2$. Third, we investigate different bursting oscillations of different cases of $b$ : for $b=0.65$, one type of bursting oscillation, namely, symmetric "homoclinic/homoclinic" bursting, is created when $c$ slow-varying passes through two homoclinic bifurcation points; for $b=1.2$, symmetric "fold/Hopf" is created when $c$ slow-varying passes through twofold bifurcation points and two Hopf bifurcation points; for $b=2$, two types of bursting oscillations, namely, symmetric "fold/fold" bursting when $c$ slow-varying passes through only two fold bifurcation points, and symmetric "Hopf/Hopf" bursting via "fold/fold" hysteresis loop is created when $c$ slow-varying passes through twofold bifurcation points and two Hopf bifurcation points. In a word, these four bursting oscillations are created due to the coexistence of different attractors. Fourth, we introduce two analysis strategies, specifically the transformed phase portrait and the overlap of the phase portrait related to $c$ with the bifurcation diagram, to explore the bursting oscillation mechanism in terms of the bifurcations and equilibria structures. Last of all, we find that the bursting oscillation can be easily controlled by the amplitude and frequency of the slow excitation. 


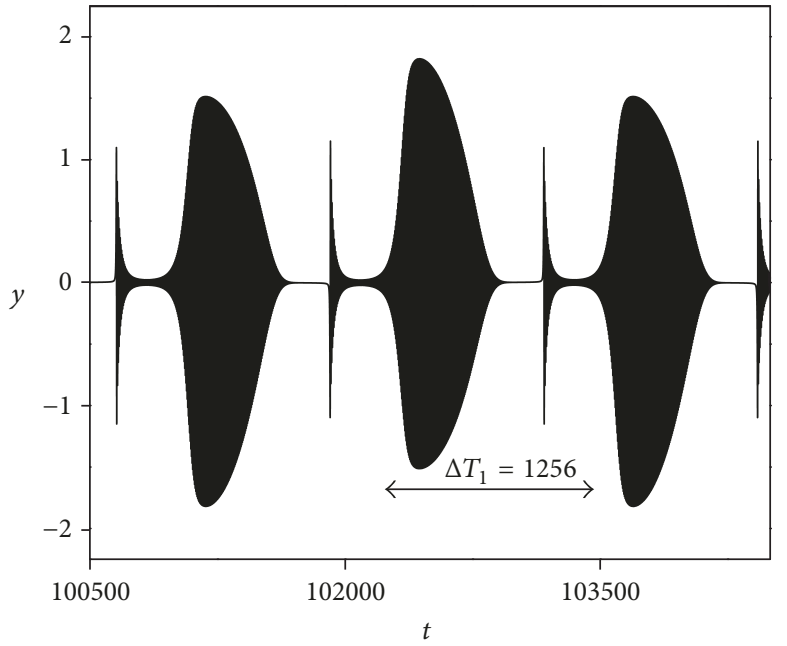

(a)

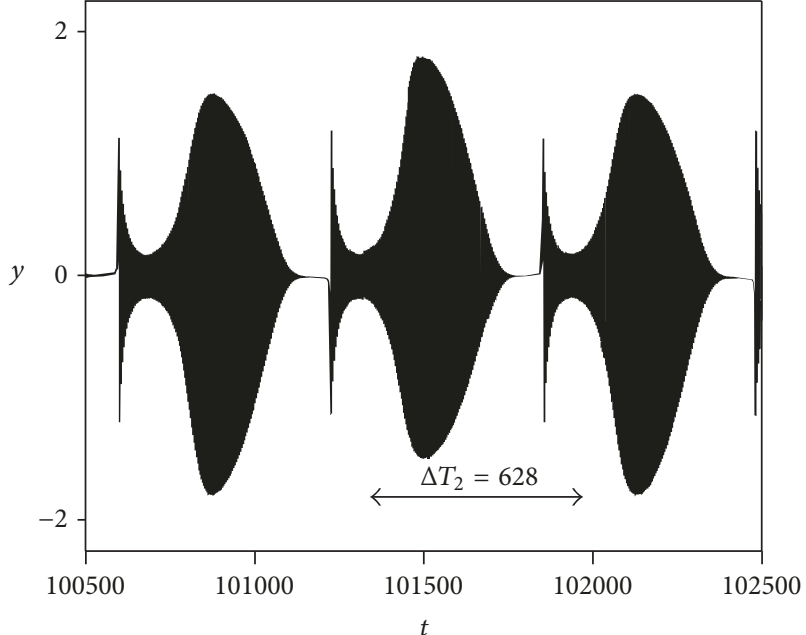

(b)

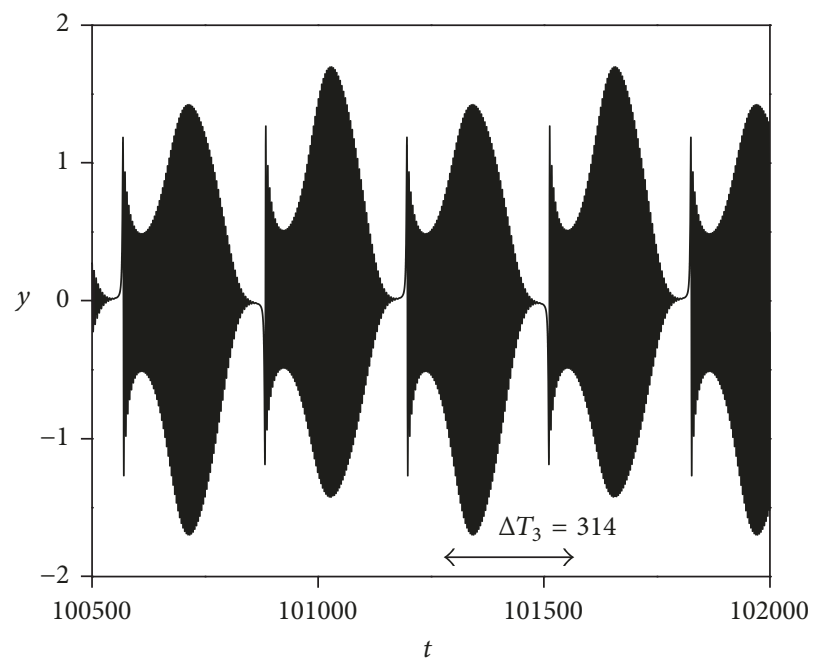

(c)

FIGURE 8: Numerical results of the time interval between two adjacent spikes of bursting oscillations corresponding to Figure 7 for the slow excitation frequency for $\omega_{1}=0.0025, \omega_{2}=0.005$, and $\omega_{3}=0.01$, respectively. (a) $\Delta T_{1} \approx 1256$; (b) $\Delta T_{2} \approx 628$; and (c) $\Delta T_{3} \approx 314$.

\section{Disclosure}

The relevant content of this article has been presented as a poster titled "Routes to Bursting Oscillations in ShimizuMorioka System with Slow-Varying Periodic Excitation" at the 17th Asian Pacific Vibration Conference, 2017.

\section{Conflicts of Interest}

The authors declare that there are no conflicts of interest regarding the publication of this paper.

\section{Acknowledgments}

The authors are grateful to Dr. H. L. Guo for useful discussions. This work is supported by the National Key Basic Research Program of China (Grant no. 2015CB057400) and the National Natural Science Foundation of China (Grant no. 11672201).

\section{References}

[1] Q. Bi, "The mechanism of bursting phenomena in BelousovZhabotinsky (BZ) chemical reaction with multiple time scales," Science China Technological Sciences, vol. 53, no. 3, pp. 748-760, 2010.

[2] J. L. Hudson, M. Hart, and D. Marinko, "An experimental study of multiple peak periodic and nonperiodic oscillations in the Belousov-Zhabotinskii reaction," The Journal of Chemical Physics, vol. 71, no. 4, pp. 1601-1606, 1979.

[3] S. W. Johnson, V. Seutin, and R. A. North, "Burst firing in dopamine neurons induced by N-methyl-D-aspartate: Role of electrogenic sodium pump," Science, vol. 258, no. 5082, pp. 665$667,1992$.

[4] M. Deschěnes, J. P. Roy, and M. Steriade, "Thalamic bursting mechanism: an inward slow current revealed by membrane hyperpolarization," Brain Research, vol. 239, no. 1, pp. 289-293, 1982. 
[5] R. M. Harris-Warrick and R. E. Flamm, "Multiple mechanisms of bursting in a conditional bursting neuron," The Journal of Neuroscience, vol. 7, no. 7, pp. 2113-2128, 1987.

[6] Z.-Q. Yang, "Transitions of different rhythm patterns of neuronal firing activity," Wuli Xuebao/Acta Physica Sinica, vol. 59, no. 8, pp. 5319-5324, 2010.

[7] Q. Bi, X. Chen, J. Kurths, and Z. Zhang, "Nonlinear behaviors as well as the mechanism in a piecewise-linear dynamical system with two time scales," Nonlinear Dynamics, vol. 85, no. 4, pp. 2233-2245, 2016.

[8] Q. Bi, S. Li, J. Kurths, and Z. Zhang, "The mechanism of bursting oscillations with different codimensional bifurcations and nonlinear structures," Nonlinear Dynamics, vol. 85, no. 2, pp. 993-1005, 2016.

[9] E. M. Izhikevich, "Neural excitability, spiking and bursting," International Journal of Bifurcation and Chaos, vol. 10, no. 6, pp. 1171-1266, 2000.

[10] Y. Ji and Q. S. Bi, "Bursting behavior in a non-smooth electric circuit," Physics Letters A, vol. 374, no. 13-14, pp. 1434-1439, 2010.

[11] K. Tsaneva-Atanasova, H. M. Osinga, T. Rieß, and A. Sherman, "Full system bifurcation analysis of endocrine bursting models," Journal of Theoretical Biology, vol. 264, no. 4, pp. 1133-1146, 2010.

[12] S. T. Kingni, L. Keuninckx, P. Woafo, G. Van Der Sande, and J. Danckaert, "Dissipative chaos, Shilnikov chaos and bursting oscillations in a three-dimensional autonomous system: Theory and electronic implementation," Nonlinear Dynamics, vol. 73, no. 1-2, pp. 1111-1123, 2013.

[13] H. Simo and P. Woafo, "Bursting oscillations in electromechanical systems," Mechanics Research Communications, vol. 38, no. 8, pp. 537-541, 2011.

[14] W. M. Schaffer and T. V. Bronnikova, "Peroxidase-ROS interactions," Nonlinear Dynamics, vol. 68, no. 3, pp. 413-430, 2012.

[15] X.-H. Li and Q.-S. Bi, "Cusp bursting and slow-fast analysis with two slow parameters in photosensitive belousov - Zhabotinsky reaction," Chinese Physics Letters, vol. 30, no. 7, Article ID 070503, 2013.

[16] T. Hongray and J. Balakrishnan, "Dynamics of bow-tie shaped bursting: Forced pendulum with dynamic feedback," Chaos: An Interdisciplinary Journal of Nonlinear Science, vol. 26, no. 12, Article ID 123107, 2016.

[17] A. Sherman, J. Rinzel, and J. Keizer, "Emergence of organized bursting in clusters of pancreatic $\beta$-cells by channel sharing," Biophysical Journal, vol. 54, no. 3, pp. 411-425, 1988.

[18] J. Rinzel, "Bursting oscillation in an excitable membrane model," in Ordinary and partial differential equations, B. D. Sleeman, Ed., 316, p. 304, Springer, Berlin, Germany, 1985.

[19] Q. Y. Wang, M. Aleksandra, P. Matjaz, and Q. S. Lu, "Taming desynchronized bursting with delays in the Macaque cortical network," Chinese Physics B, vol. 20, article 040504, 2011.

[20] I. Z. Kiss, L. N. Pelster, M. Wickramasinghe, and G. S. Yablonsky, "Frequency of negative differential resistance electrochemical oscillators: Theory and experiments," Physical Chemistry Chemical Physics, vol. 11, no. 27, pp. 5720-5728, 2009.

[21] X. Li, Z. D. Zhang, and Q. S. Bi, "Mechanism of bursting oscillations in non-smooth generalized Chuas circuit with two time scales," Acta Physica Sinica, vol. 62, article 20502, 2013.

[22] T. Shimizu and N. Morioka, "On the bifurcation of a symmetric limit cycle to an asymmetric one in a simple model," Physics Letters A, vol. 76, no. 3-4, pp. 201-204, 1980.

[23] P. Mandel and T. Erneux, "The slow passage through a steady bifurcation: delay and memory effects," Journal of Statistical Physics, vol. 48, no. 5-6, pp. 1059-1070, 1987. 


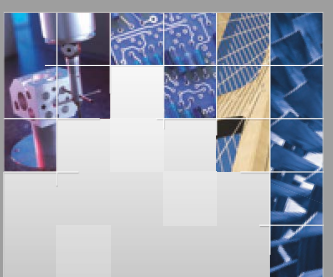

\section{Enfincering}
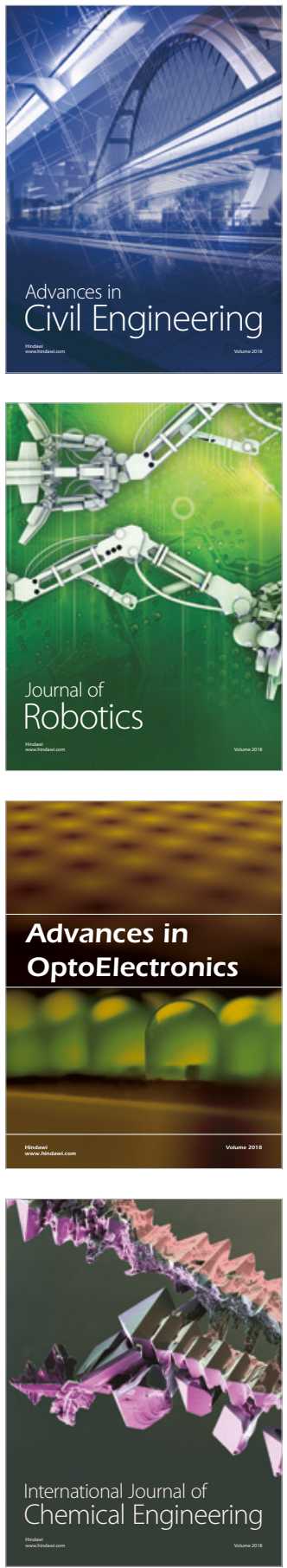

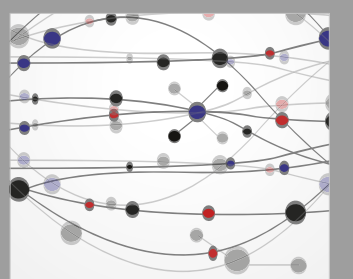

\section{Rotating \\ Machinery}

The Scientific World Journal

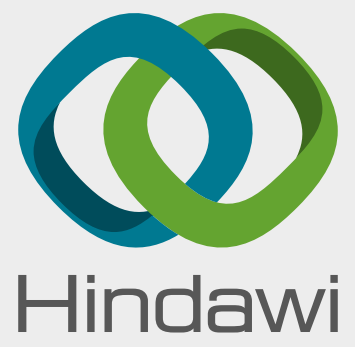

Submit your manuscripts at

www.hindawi.com
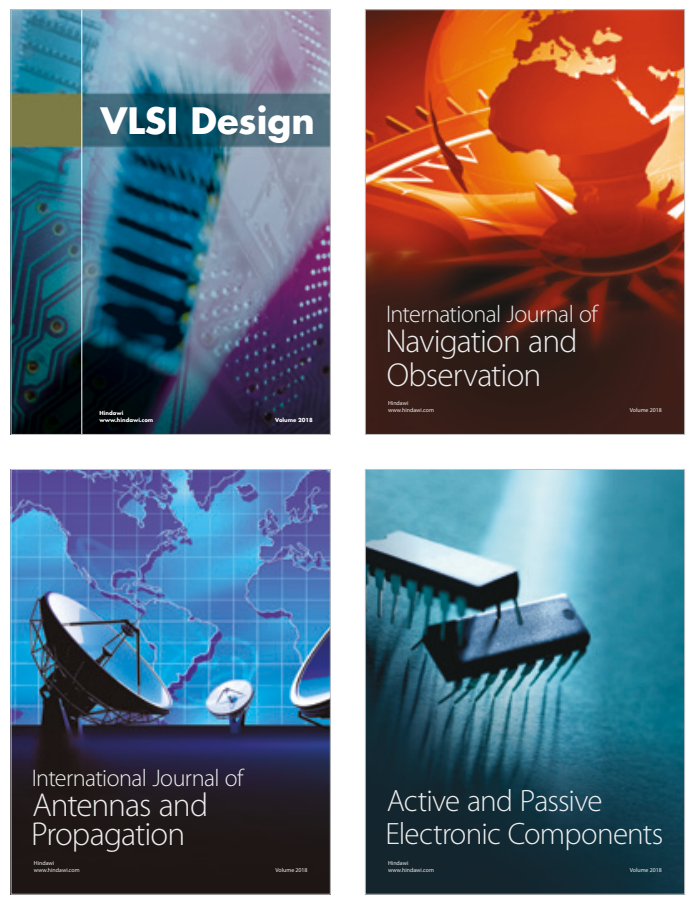
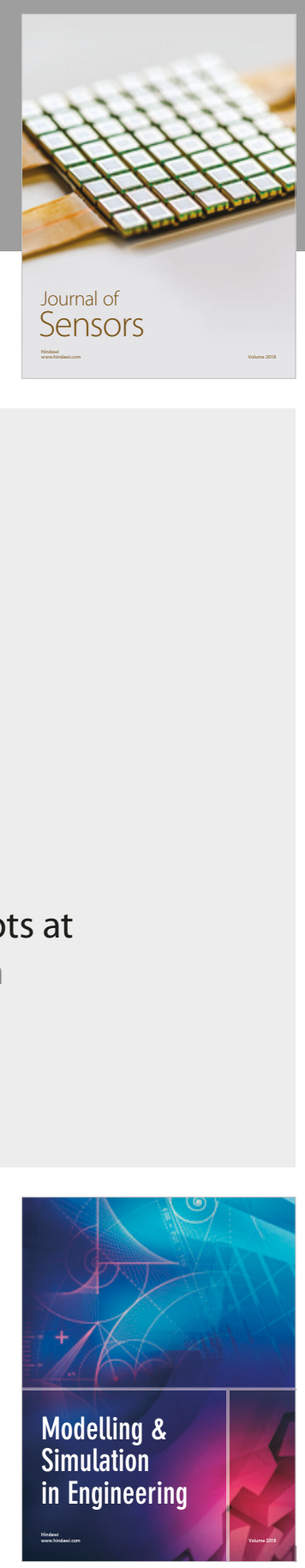

\section{Advances \\ Multimedia}
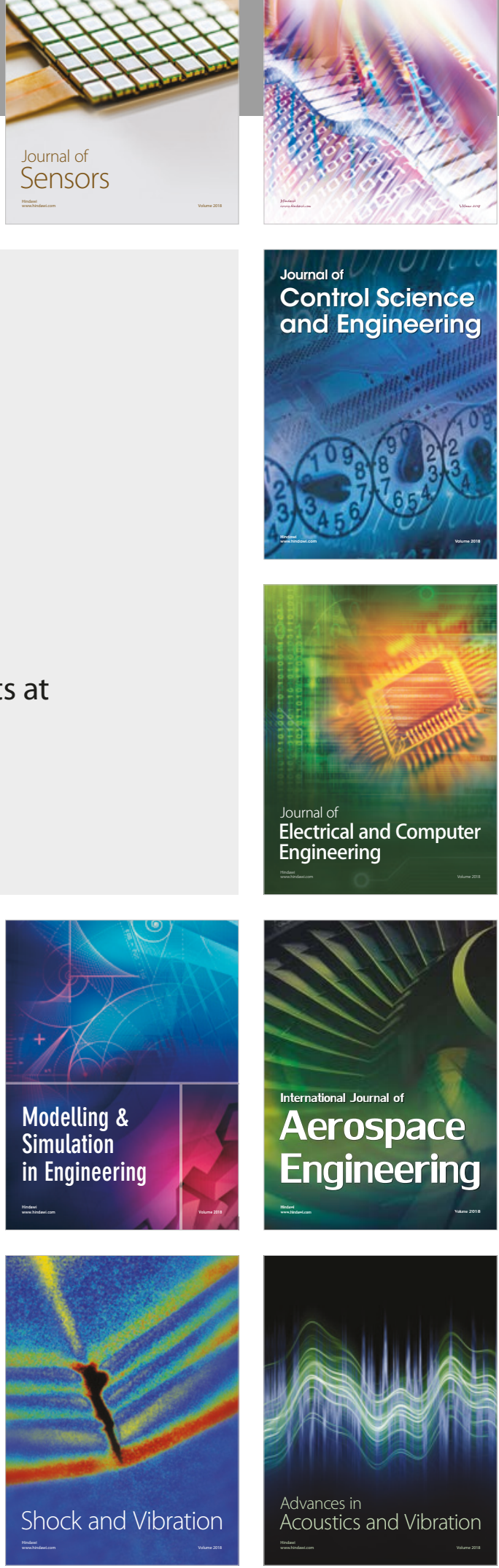